\title{
Spatially Explicit Modeling of Community Occupancy using Markov Random Field Models with Imperfect Observation: Mesocarnivores in Apostle Islands National Lakeshore
}

\author{
Yunyi Shen ${ }^{1,2 凶}$ Erik Olson $^{3}$, and Timothy R. Van Deelen ${ }^{1}$ \\ ${ }^{1}$ Department of Forest and Wildlife Ecology, UW-Madison, 1630 Linden Dr. Madison WI, USA, 53706; ${ }^{2}$ Department of Statistics, UW-Madison, 1300 University Ave \\ Madison WI, USA, 53706; ${ }^{3}$ Department of Natural Resources, Northland College, 1411 Ellis Avenue, Ashland, WI, USA 54806
}

\begin{abstract}
How species organize spatially is one of ecology's most attractive questions. Multiple theories have been advanced and various models developed to account for environment, interactions among species, and spatial drivers. However, relative importance comparisons of explanatory phenomena generally are neglected in these analyses. We developed a spatially explicit community occupancy model based on Markov random fields that accounts for spatial auto-correlation and interspecific interactions in occupancy while also accounting for interspecific interaction in detection. Simulations demonstrated that the model can distinguish different mechanisms of environmental sorting, such as competition and spatial-autocorrelation. We applied our model to camera trap data from a fisher(Pekania pennanti)-marten(Martes americana) and coyote(Canis latrans)-fox(Vulpes vulpes) system in Apostle Island National Lakeshore (Wisconsin, USA). Model results indicated that the observed partitioning pattern between marten and fisher distributions could be explained best by a flipped mainland-island source-sink pattern rather than by competition. For the coyote-fox system, we determined that, in addition to a mainland-island source-sink pattern, there was a positive association between fox and coyote that deserved further study. Our model could be applied readily to other landscapes not restricted to islands, therefore enhancing our understanding to species coexistence patterns.
\end{abstract}

Species Coexistence | Occupancy Model | Markov Random Field | Fisher | Marten | Coyote | Red fox

Correspondence: yyshen@pku.edu.cn

\section{Introduction}

Drivers of species distributions and community structures are among the most important questions in ecology. Classical theories include niche theory (Hutchinson, 1957) and Lotka-Voterra models (Lotka, 1910; Volterra, 1928). These theories concentrated on interactions between species and their environments (hearafter: environment sorting where environmental filters select for species with certain characteristics and allow them to coexist) and interaction among species (hearafter:interspecies interactions, e.g. competition) while generally ignoring the spatial arrangement of habitat patches. In contrast, MacArthur and Wilson (2001) emphasized the importance of random patch-level colonization and extinction probabilities in forming species richness patterns. This idea was further developed by Hubbell's neutral theory on community assemblage (Hubbell (2001), see Volkov et al. (2003) for a review). Meta-population modelling is another example of spatially explicit theory, which emphasized the importance of dispersal (Hanski, 1983). This paradigm emphasized importance of geographic arrangement of habitat patches (spatial processes, e.g. spatial auto-correlations) in determining the distributions of species. However, recent research suggested that communities reflect both species- and patch-level drivers. Leibold et al. (2004) extended meta-population models to examine community assembly, while accounting for spatial and natural history processes concurrently. Yet, the relative importance of natural history processes (environmental sorting and species interactions) and spatial processes remained unclear in most communities and unaddressed in most analyses. Researches has examined plant communities (e.g. Lasky et al. (2017)), marine systems (e.g. Shurin et al. (2009); Göthe et al. (2013); Meyer (2017)), and microbial systems separate spatial, environmental and interspecific interactions in both experimental and natural communities (see Logue et al. (2011) for a review). These studies suggested that a gradient from almost fully spatial-driven to almost fully environment/interaction-driven patterns in community assemblage.

Due to recent advancements in camera-trap technology, ecologists can generate vast quantities of detection/non-detection data to infer presence-absence (P/A) of moderate to large-sized animals. However, imperfect detection (i.e. false absence due to imperfect sampling) remains a challenge (Kéry and Schmidt, 2008). Occupancy modeling (MacKenzie et al., 2003) addresses imperfect detection by modeling a detection process explicitly and estimating detection rates from repeated sampling. Following a basic idea of modeling occupancy and detection hierarchically, one can build various occupancy-like models based on the idea that observations are samples taken from detection distributions conditioned on unobserved latent true patterns that follow other characteristic distributions. To understand both environmental sorting and inter-specific interactions, researchers proposed use of multispecies occupancy models of two kinds. The occupancy model developed by Rota et al. (2016) used a multinomial-logistic regression which estimated different predictors for different coexistence patterns, while Kéry and Royle (2008) used a hierarchical structure to model species interactions that can be viewed as a Bayes network (Koller and Friedman, 2009). These techniques facilitated research on assembly of animal communities in island systems and other landscapes. However, neither Kéry and Royle (2008) nor Koller and Friedman (2009) can model interactions of species and spatial processes simultaneously and explicitly in order to understand their relative importance (Cottenie, 2005; Dray et al., 2006). This, because too many possible patterns make multi-logistic modeling intractable as in Rota et al. (2016) and the spatial correlation cannot be represented using directed graphs as in Kéry and Royle (2008). 
bioRxiv preprint doi: https://doi.org/10.1101/2020.08.05.238774; this version posted March 31, 2021. The copyright holder for this preprint (which was not certified by peer review) is the author/funder, who has granted bioRxiv a license to display the preprint in perpetuity. It is made available under aCC-BY-ND 4.0 International license.

Probabilistic Graph Modeling (PGMs, Koller and Friedman (2009)) provides a general framework for modeling systems with unspecified dependence structures (e.g. competition between species, spatial auto-correlation between sites). Markov Random Field modeling (MRF) is a kind of PGM that defines joint distributions of sets of random variables linked by non-directed graphics (Vanmarcke, 2010; Cressie, 1992). MRF has long been used to model spatial correlations in ecology and agriculture, e.g. in spatial ecology (Hughes et al., 2011; Hepler et al., 2018), as well as temporal analysis (Zhu et al., 2005) and interspecific interactions (Harris, 2016). It was also widely used for modeling networks in social systems (West et al., 2014), genetic associations (Wei and Li, 2007), as well as competing species (Harris, 2016). MRF models with imperfect observations were also explored in image reconstruction contexts (Chalmond, 1989; Ibáñez and Simó, 2003). In contrast to Bayes networks Kéry and Schmidt (2008), MRF models allowed cycles in the interaction network, i.e. there was no need for a root species that all other species were conditioned on, and allowed species $A$ to interact with $B, B$ to interact with $C$ and $C$ to interact back with $A$, meanwhile, MRF was the classical model for modeling spatial autocorrelations. Thus MRF-based occupancy-like models could be an alternative for joint modeling of environmental, interspecific interaction, and spatial drivers. Our objective was to develop a model to capture spatial auto-correlation and interspecific interactions while controlling for environmental predictors and, consequently, understanding the drivers of distributions of close competitor species in the Apostle Islands National Lakeshore (APIS, Wisconsin, USA).

We focus on two pairs of plausibly competing species: fisher(Pekania pennanti)-marten(Martes americana) (fisher-marten system) and coyote(Canis latrans)-red fox(Vulpes vulpes) (coyote-fox system). In APIS, 30\% of sites with fisher detections also had marten detections and $15 \%$ of sites that had marten detections also had fisher detections (2014-2017), in contrast, $64 \%$ of sites that had red fox detections also had coyote detections and while $28 \%$ of sites that had coyote detections also had red fox detections (Fig.1). In niche-based theories, a partition pattern like fisher and marten could be understood as spatial niche partitioning due to competition, while coexistence could be achieved by partitioning other niche dimensions such as time. In these theories, competition was a factor promoting the partition pattern. In dispersal-related theories, species were more likely to exist close to the source of dispersing individuals which is usually a stable mainland population. If species were independent, they would coexist on islands closer to mainland with higher probability subject to dispersal capability. Since we observe a spatially partitioned pattern for fisher-marten, it may be explained by competition or different source-sink dynamics (spatial processes). Importantly, we observed that marten tended to occur on more distant islands that might indicate a "flipped" mainland-island pattern opposite the prediction of the mainland as source. For coyote and fox, coexistence at islands closer to the mainland, could be explained by dispersal from the mainland. Yet, after accounting for such potential spatial processes, we are interested also in determining if these two species associate or compete (dominant competitor excludes sub-dominant). We were unable to obtain detailed environmental predictors in this relatively small and homogeneous area, hence, the only "environmental" predictor considered here was distance to mainland (negative exponential transformed) which should be considered as part of a spatial process.

For the fisher-marten system, we pose two working hypothesis for the observed pattern:

1) Distribution of both species on the islands reflects similar mainland-distance dependencies (spatial effects). While separation at the site level is due to competition (interaction effects)

2) Distribution of both species on the islands reflects differing mainland-distance dependencies but shows minor competition at the site level.

For the coyote-fox system, we pose two working hypothesis for the observed pattern:

1) Distribution of both species in the islands reflects spatial drivers. Coexistence is facilitated by separation in time.

2) Trophic position and life-history drives distribution (foxes avoid coyotes at the site level), while spatial effects at the island level are minor.
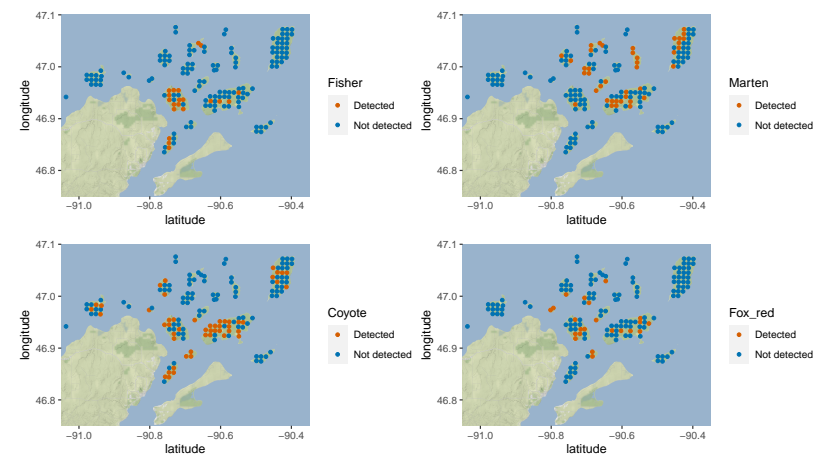

Figure 1. Detection of 4 target species on the islands, blue: detected, red: not detected, dots represent camera locations on the APIS

\section{Methods}

Study Area. Apostle Island National Lake Shore is located on the southwest shore of Lake Superior (USA) and lies in the transition zone between temperate and boreal forest regions. APIS is distinct from tropical islands (where much research on community assembly has occurred) because of severe winters and relatively low primary productivity. Ten species of native carnivores were detected during 2014-2017 (Allen et al., 2017) (Fig.1). How these species coexist and how richness differs between islands is a fundamental question for understanding community dynamics in temperate island systems.

Camera trapping surveys. During 2014-2017, APIS staff and collaborators conducted camera-trapping surveys to determine distributions and relative abundances of mammal carnivores in the National Lakeshore (Allen et al., 2017). Twenty-one of 22 islands which make up the archipelago were surveyed using a $1 \mathrm{~km}^{2}$ lattice (grid) sampling frame. Within each grid cell there was one camera trap. Scent lures were placed at half of the camera stations at deployment and rotated to the remaining stations during mid-deployment camera checks. Since all islands could not be monitored at the same time, though substantial overlap did occur, we assume the underlying distribution of species did not change during the 3-year survey period. We divided surveys into 60-day blocks to create repeat observations. 
bioRxiv preprint doi: https://doi.org/10.1101/2020.08.05.238774; this version posted March 31, 2021. The copyright holder for this preprint (which was not certified by peer review) is the author/funder, who has granted bioRxiv a license to display the preprint in perpetuity. It is made available under aCC-BY-ND 4.0 International license.

Spatial Explicit Community Occupancy Analysis using Binary Markov Random Field Model. We used Ising model (Ising, 1925), a kind of Markov Random Field (MRF) model whose responses are all binary, to model the distribution of competing species in a spatially-explicit manner. Coding of latent true occupancy status followed the convention in network science, i.e. +1 for presence and -1 for absence. This symmetric coding was more conventional in physics but less so in ecology. Models with a centering term enabled modeling of the "large scale" response due to environmental predictors (centered autologistic models Hughes et al. (2011)) which tried to detect auto-correlation in the residuals of a large scale response due to environmental drivers were used in ecology. However theoretical studies with this model by Wolters (2017) suggested better performance of a symmetrical coding rather than centering the model when associations (e.g. competition, spatial autocorrelations) were expected. Additionally, centered models predicted non-linear relationships between strength of association and log odds of two species coexisting or co-absenting while symmetrical coding did not have this problem. Further, symmetrical coding avoided the cross product between different terms (i.e. environment and species interactions) in the negative potential function (log probability mass function (pmf) up to a constant difference) of this model (Koller and Friedman, 2009) which helped us evaluate relative contributions of different mechanisms. In addition, parameters of a symmetrical-coding model had better conditional interpretation, e.g. regression coefficient $\beta$ was the conditional log odds of presence given all other sites and species. This property was important in understanding the strength of different mechanisms (Blanchet et al. (2020), argument 2).

To make comparisons between environmental sorting, spatial process and interspecific interactions drivers in shaping species' spatial distributions, three components were considered simultaneously but separately in the graph associated with the joint distribution: 1) a linear predictor calculated from environmental covariates 2) a nearest neighborhood spatial autocorrelation at camera-site level (site level hereafter,Hepler et al. (2018)) within and among islands and 3) local species associations at site level (We assume that partial associations reflect interactions, similar to Harris (2016)). We denote the design matrix for environmental covariates as $\mathbf{X}$ and responses of certain species $k$ ( $k=$ $1,2 . ., w)$ to environment $\mathbf{X}$ as $\beta_{k}$. Further in this case study, due to the different nature of site linkages within and across islands, inter-island and intra-island correlations were modeled separately. We denote the strength parameters of these two correlations as $\eta^{e x}$ and $\eta^{i n}$, and known adjacency matrix $\mathbf{D}^{e x}$, $\mathbf{D}^{i n}$ (eqn.1). Mainland-island with linkage matrix $D^{m l}$ shares the same strength of inter-island spatial autocorrelation in this study. We denote the presence and absence vector of species $k$ on the landscape as $\mathbf{Z}_{k}$. We denote the transpose of $\mathbf{Z}_{k}$ as $\mathbf{Z}_{k}^{T}$ Thus, the joint distribution of all species at all sites has form:

$$
\begin{aligned}
P\left(\mathbf{Z}_{1}, \ldots, \mathbf{Z}_{w} \mid \theta\right) \propto \exp \left[\sum_{k=1}^{w}\right. & \left(\left(\mathbf{X} \beta_{k}\right)^{T} \mathbf{Z}_{k}\right. \\
& +\eta_{k}^{e x} \mathbf{Z}_{k}^{T} \mathbf{D}^{m l} \\
& +\frac{1}{2} \eta_{k}^{i n} \mathbf{Z}_{k}^{T} \mathbf{D}^{i n} \mathbf{Z}_{k} \\
& +\frac{1}{2} \eta_{k}^{e x} \mathbf{Z}_{k}^{T} \mathbf{D}^{e x} \mathbf{Z}_{k} \\
& \left.\left.+\sum_{l>k} \gamma_{l k} \mathbf{Z}_{k}^{T} \mathbf{Z}_{l}\right)\right]
\end{aligned}
$$

For detailed meaning of parameters see Table.1. We use $\theta$ as an abbreviation of all parameters in a conditional probability. Note that the first term accounts for an environment response (mainland-island effect), the second accounts for mainland-island process (as a special environment predictor, mainland-island spatial effect), the third term accounts for intra-island spatial auto-correlations (spatial effect), the fourth term accounts for inter-island spatial auto-correlations (and can be other types of auto-correlations) and the last term accounts for all inter-specific interactions between species $k$ and species $l$ while $\gamma_{l k}$ is the strength of association between the two species. In the mainland-island setting, we assumed that there were no inter-island spatial auto correlation so $\mathbf{D}^{e x}$ has all 0 as its entries.

Accounting for Imperfect Detection and Short-term Interactions. Following the logic of occupancy-like modeling (MacKenzie et al., 2003), we model observed detection/non-detection as repeated samples from a detection process. Associations in short-term detection can also be informative about species interaction. We further assume that the interspecific interactions are local (i.e. no spatial auto correlations considered in the detection process). We used another binary MRF (Ising model) conditioned on occupancy status of a species to model the detection process. In total, there were two binary MRF models: 1) latent occupancy, and 2) detection conditioned on occupancy. Only species occupying a certain site will be included in the detection MRF and species not occupying will have probability of non-detection of 1 . Formally, we denote $y_{k i j}$ as species $k$ 's detection status at site $i$ during period $j$. The likelihood function at site $i$ and detection repeat $j$ is given by eqn.2.

$$
\begin{aligned}
& P\left(y_{1 i j}, y_{2 i j}, \ldots \mid Z_{1 i}, Z_{2 i}, \ldots, \theta\right) \propto \\
& \quad \begin{array}{l}
\exp \left(\sum _ { k = 1 } ^ { w } \left[\mathbf{X}_{i j}^{\text {det }} \beta_{k}^{\text {det }} y_{k i j} I_{Z_{k i}=1}\right.\right. \\
\left.\left.\quad+\sum_{l>k} \gamma_{l k}^{\text {det }} y_{k i j} y_{l i j} I_{Z_{k i}=1} I_{Z_{l i}=1}\right]\right)
\end{array}
\end{aligned}
$$

We use $\theta$ as an abbreviation of all other parameters in the conditional probability. $I_{\{\}}$is the indicator function and $I_{Z_{l i}=1}=1$ only if $Z_{l i}=1$ and $I_{Z_{l i}=1}=0$ otherwise. The indicator function will "knock out" the species from detection interaction if it was not occupying that site. The reasoning behind this knocking out was that we assume that non-detection was caused by absence of a species and thus should be understood as a do-calculus (Pearl, 1995) rather than conditioning. Unlike the occupancy part, this conditional likelihood function is tractable for reasonable numbers of species (e.g. $<10)$ because of the relatively small size of the underlying graph. The joint likelihood function of the whole 
bioRxiv preprint doi: https://doi.org/10.1101/2020.08.05.238774; this version posted March 31, 2021. The copyright holder for this preprint (which was not certified by peer review) is the author/funder, who has granted bioRxiv a license to display the preprint in perpetuity. It is made available under aCC-BY-ND 4.0 International license.

detection history, conditioned on occupancy was the product of each site and period. The joint (unnormalized) likelihood function of observed detection data then can be calculated by multiplying eqn.1 and eqn.2. The latent $\mathbf{Z}$ 's can be estimated similarly with unknown parameters.

Priors were set to be vague normal distributions due to the relatively small number of repeats and lack of environmental variation in our APIS case study. We put a normal prior with variance 0.1 on intercept of detection (0.95 HDR for detection rate: $[0.22,0.78])$ as part of our assumptions. Again this was not necessary for the model per se (as seen in simulation), but part of the case study. Sensitivity analysis on this part was also conducted. Posterior distributions were simulated through a Markov chain Monte Carlo (MCMC) algorithm (Hastings, 1970). To overcome the double-intractable nature of the posterior (Murray et al., 2012; Møller et al., 2006), we followed the single parameter change method proposed by (Murray et al., 2012). We calculate a p-value level that credible interval $(\mathrm{Cl})$ spans 0 . The full description of the algorithm used can be found in Appendix.S2. Diagnostic evaluation of MCMC results were done using $\mathrm{R}$ package coda (Plummer et al., 2006).

Selection Between Stepping-Stone and Mainland-Island Model. We compared two general models for spatial auto-correlation between islands in this study.

1) A stepping-stone model assumed that sites at the edge of an island can be a neighbor to sites on another island in a MRF sense. We assign this linkage using Delaunay triangulation (Okabe et al. (2009), Fig.S1). Strength of correlation was assumed to decay exponentially through the normalized distance (Shurin et al., 2009). Sites on the closest islands have linkage to the mainland and the log odds of having species occupying such site decay exponentially through the normalized distance to mainland (Shurin et al., 2009).

2) A mainland-island model assumed that sites on different islands were conditionally independent given their distance to mainland, the log odds of having species occupying a site decayed exponentially through the normalized distance to mainland (Shurin et al., 2009).

Bayes Factor (BF), a Bayesian generalization of a Likelihood ratio test, can be used for model selection (Gelman et al., 2013). We assume two models are equally plausible and thus Bayes factor can be understand as the ratio between posterior probabilities of the two models. We can calculate the posterior predictive distribution of data following Raftery et al. (2006). One obstacle to using BF in this model is the intractable likelihood function preventing us from directly calculating the predictive probability of each model by calculating the likelihood function during the posterior sampling. However we could follow Descombes et al. (1999) in calculating the likelihood function by sampling augmentation variables (Appendix. S2). Since the ratio is also estimated, robustness diagnostics following Descombes et al. (1999) could be conducted.

Evaluating Contribution of different Drivers. Different processes can drive degree of spatial partitioning among species, e.g. environmental sorting can promotes coexistence when species need similar resources (Grinnell, 1917; Saporetti-Junior et al., 2012) while competition promotes partitioning (Tilman, 1985; Gastauer and Meira-Neto, 2014). A different question, compared with the strength and direction of each process was: which driver makes the observed pattern likely? For instance, is it possible that while species $\mathrm{A}$ and $\mathrm{B}$ compete, we observe that they still coexist because of environmental sorting? In this case, we may argue that environmental sorting had a larger contribution to the observed (coexistence) pattern.

We propose to use negative potential functions (a.k.a. Hamiltonian functions in our specific setting from Cipra (1987) eqn.1.1, Osogami (2017); Pfeuty (1970)) as statistics to evaluate the contributions of each driver on the observed pattern as drivers are modeled explicitly in the proposed model. In our setting the Hamiltonian function is the log of the right hand side of eqn.1. These types of statistics were used to quantify "fitness landscapes" of amino acid interactions in protein systems (Levy et al., 2017; Shekhar et al., 2013; Ferguson et al., 2013; Morcos et al., 2014) with many other applications in quantifying stability of interactions (Ezaki et al., 2017; Becker and Karplus, 1997; Cipra, 1987).

Statistically, a negative potential function can be viewed as a fitness score defined by log probability mass function (pmf) up to a constant difference. Patterns with higher scores had higher probabilities of occurrence (Levy et al., 2017). In the model specification, we used several distinct terms to account for different ecological processes and we can use the score of each of the terms to evaluate the relative contribution of each process to the observed pattern. For instance, $\gamma_{l k} \mathbf{Z}_{k}^{T} \mathbf{Z}_{l}$ is the score for interspecific interaction between species $l$ and $k$ where $\gamma_{l k}$ is the strength of the interaction and $\mathbf{Z}_{k}$ is the vector of the occupancy of species $k$. The score is an "accumulated" strength through occupancy. Note the score assesses the probability mass of the observed pattern, i.e. a high positive contribution to the negative potential function means the corresponding term made the probability mass on the observed pattern high. The question to be answered here is whether a certain process (e.g. spatial) had large contribution to the observed pattern as we argued above. The strength of a process is different from its contribution to the observed pattern, a different aspect of the hypothesis. Posterior distributions for different terms of negative potential functions were calculated using posterior samples of latent occupancy status and model parameters. All implementations were in R ( $R$ Core Team, 2019) and $C_{++}$(Eddelbuettel and Sanderson, 2014; Eddelbuettel and François, 2011). Implementations can be found on the author's github here.

Simulations. Simulations were conducted using the same spatial arrangement of APIS' camera trapping grids, as well as regular grids sized $10 \times 10,15 \times 15,20 \times 20$ and $25 \times$ 25. On APIS' camera grids, we tested three mechanisms: 1) Competition, 2) Non-interaction and 3) Sorting. In competition simulations, two species had the same reaction to environment (or distance to mainland for APIS) and a negative association. In no-interaction simulations, two species had opposing reactions to environment or distance to mainland (represent niche difference/flipped source-sink) and no association. In sorting simulation, two species had the same but relative weak reaction to environment or mainland distance and a positive association. There were spatial auto-correlations on all mechanisms. On regular grids, we tested two species on a random draw landscape with one environmental predictor (note that we did not randomize this environment) while on APIS, we used distance to mainland as the predictor since there was a lack of environmental variation. For detailed simulation settings see Table.S1 and Table. S2. 


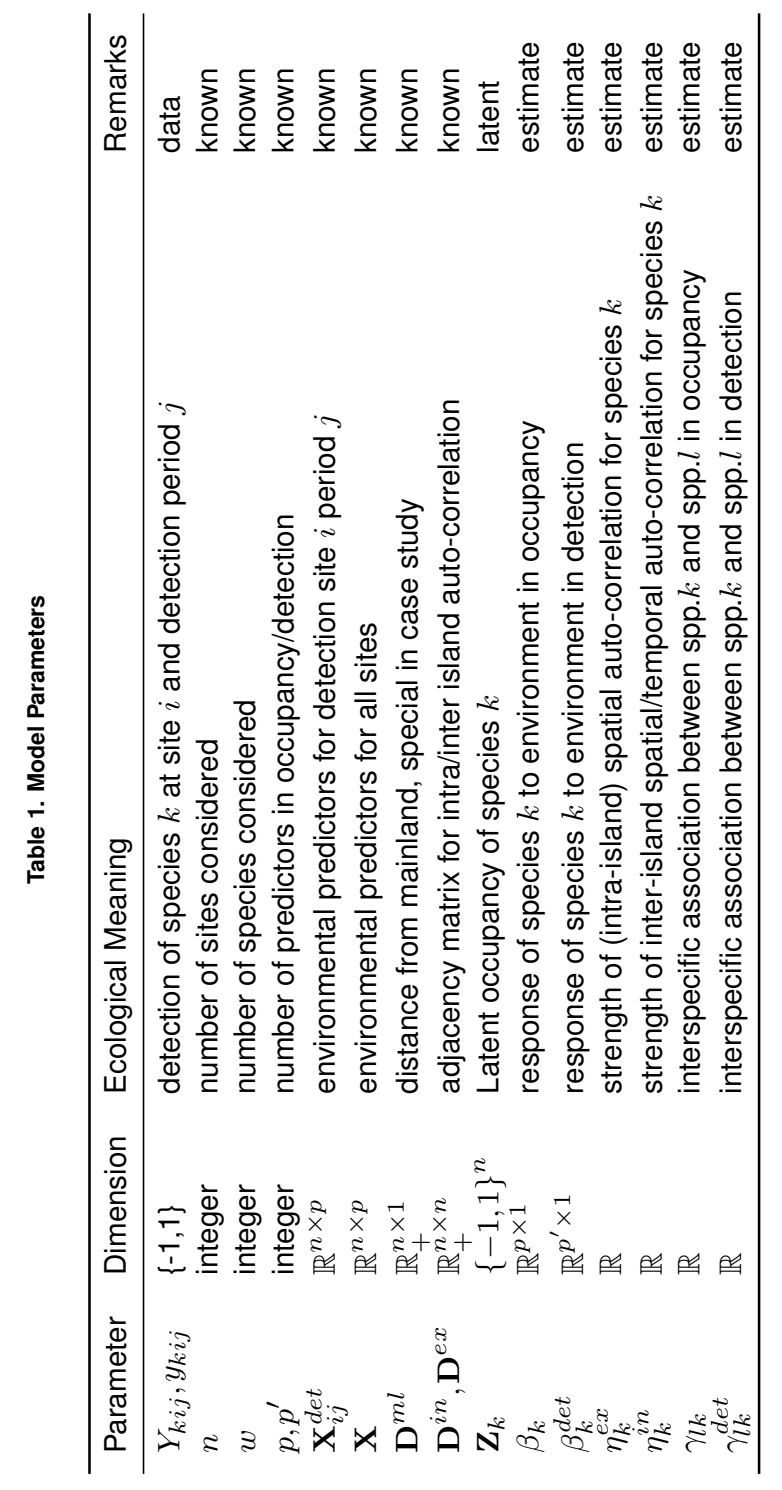




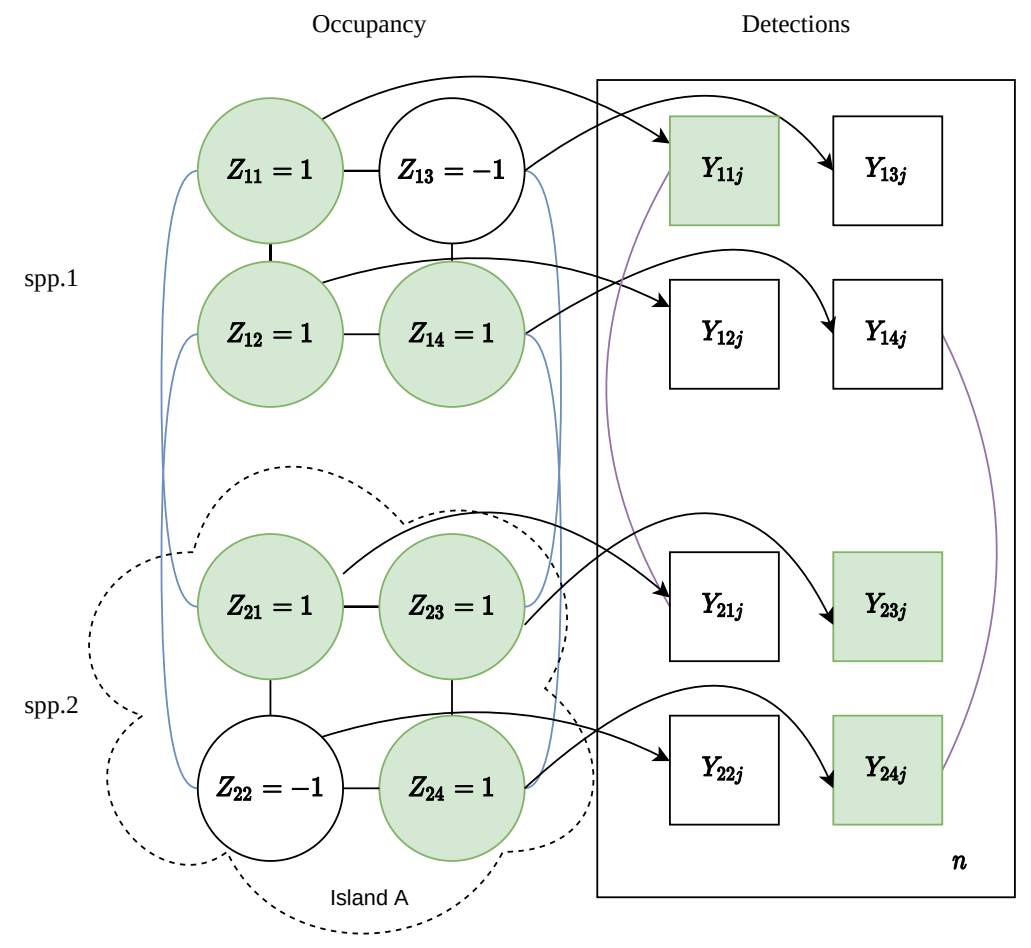

Figure 2. Dependence structure on single island, with two species. Squares indicate observed detections while circles indicate latent occupancy, green shows 1 or detection/occupancy, connections between nodes indicates conditional dependence. Upper parts showed species 1 while lower was for species 2 , note that only if two species coexist can there be an edge between the detection

In simulations, all parameters had vague priors.

Posterior Predictive Checks. To check for systematic discrepancies between data and model predictions, we performed posterior predictive checks (Gelman et al., 1996; Lynch and Western, 2004; Gelman et al., 2013) using 6 statistics for the APIS case-study. They were: 1) the frequency of detection for fisher and marten and 2) frequency of detection for coyote and fox, this represented how often we see the species; 3) mean overall sites that had at least one detection for fisher and marten or 4) for coyote and fox, this represented the naive occupancy; 5) correlations between naive occupancy in each system (fisher-marten or coyote-fox), in which naive occupancy was 1 if species was ever seen at that site and -1 otherwise; 6 ) number of sites with detection of both species from either of our species-systems.

In total 2,000 posterior predictive detection histories with the same time frame as the original APIS data were sampled and posterior predictive $p$-values for each statistic were calculated. Small $p$-values indicated variations the model failed to capture.

\section{Results}

Simulations. Differences between posterior median estimates and true parameter values in 100 simulated datasets were evaluated (Fig.3). In all cases 0 was contained in the middle $50 \%$ quantile, suggesting that we generally could recover parameter values using regression models. However, we were conservative regarding spatial auto-correlations (posterior medians were less than true parameter values in some cases) due to relatively a small number of grids, as also shown in Hughes et al. (2011). In light of this, to better estimate spatial auto-correlation we need either a larger grid or a greater number of repeats.
Fisher-Marten System. A total of $3 \times 10^{6}$ samples were drawn after $5 \times 10^{4}$ burn in and thinned by 300 . Diagnostics of MCMC showed sufficient mixing of the chains (Fig.S2), suggesting that the MCMC algorithm can approximate the posterior distribution of parameters. $\log _{10}$ Bayes factors for mainland-island models and stepping stone models were estimated to be 4.26 , (i.e. the posterior probability of mainland-island model was $10^{4}$ higher than the posterior probability of stepping stone model) hence data decisively supports mainland-island rather than the stepping stone model following the recommended cutoff of 2 (Kass and Raftery, 1995). This result suggested that the spatial pattern of the system can be explained better by a mainland-island model rather than a stepping stone model. Further analysis will be based on the Mainland-Island model.

Table.2 shows the posterior estimates of model parameters of interest for the mainland-island model of the fisher-marten system. We detected a significant positive distance dependency (positive means higher chance to occupy a closer island here because distance is negative exponentially transformed) in fisher $\left(\eta^{e x}=2.479, C I=[0.976,4.744]\right)$ and a negative distance dependency in marten occupancy $\left(\eta^{e x}=\right.$ $-0.789, C I=[-1.907,-0.0558])$.

However, we did not detect significant association between these species in either occupancy or detection $(C I=[-0.215,0.393], \quad[-0.326,0.322]) . \quad$ Marten showed an intra-island spatial auto-correlation 0.260 $C I=[0.0551,0.444])$. This result supported the second hypothesis that fisher and marten had a "flipped" mainland-island pattern whereby fisher had a higher chance of occupying closer islands while martens had a higher probability of occupying more remote islands independently of possible competition.

As discussed in our methods, the strength of a certain 


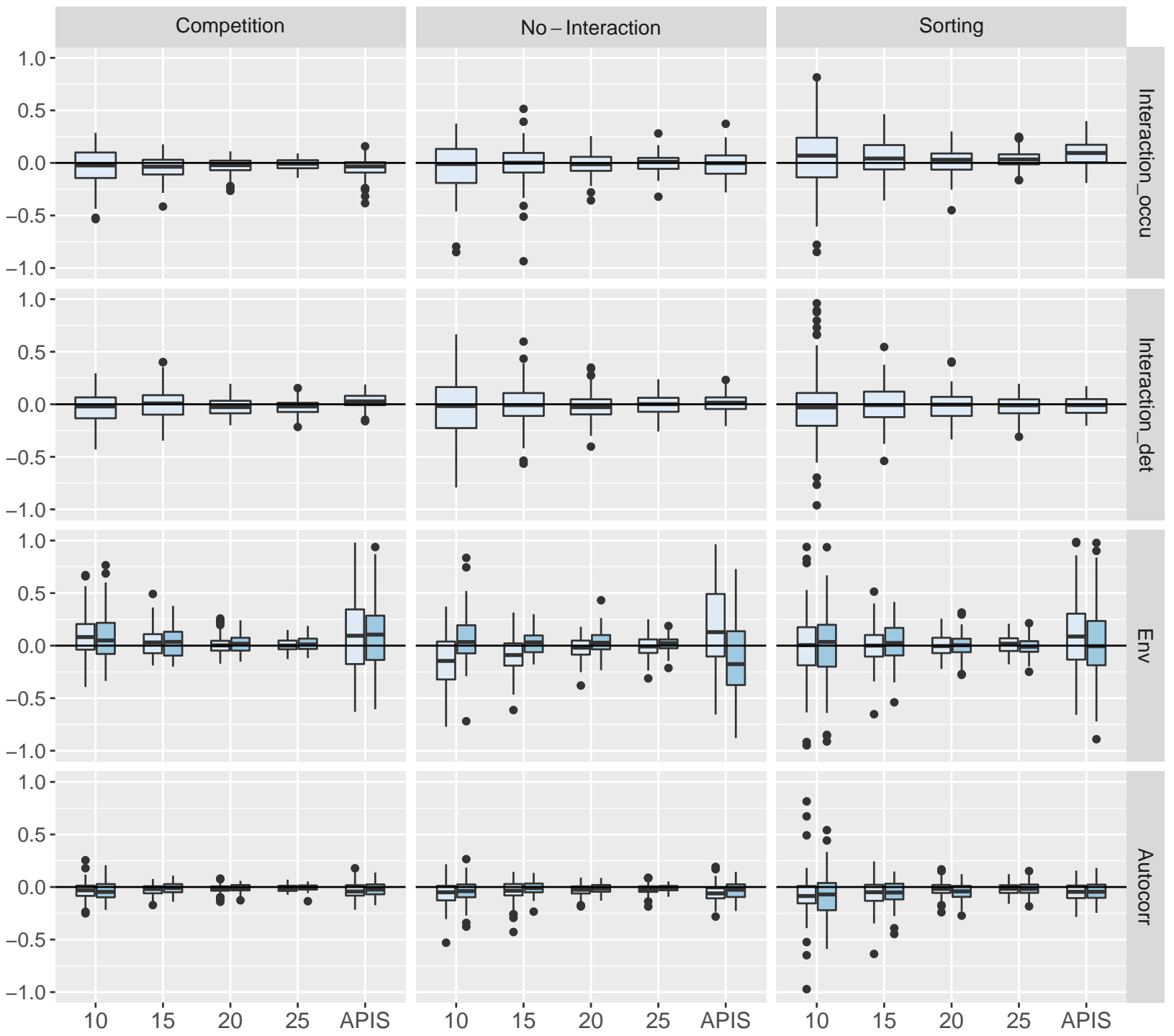

Figure 3. Differences between posterior medians and true parameter values of $\mathbf{1 0 0}$ simulated data sets. First column: Competition, species had same environment dependency and negative interspecific interaction, Second column: No interaction, species had different environment dependency and no interaction, Third column: Sorting, species had same environment dependency and positive interspecific interaction. Rows correspond to parameters estimated in the model. First row: Interaction in occupancy, Second row: Interaction in detection, Third row: Reaction to environment, Fourth row: Spatial autocorrelation. X axis was the size of lattice or APIS (155 grids). Shading of boxes indicates each of the two "species" simulated

Table 2. Posterior estimation of model parameters in Fisher-Marten mainland-island system. $\eta^{e x}$ represented the distance dependency, $\eta^{e x}>0$ meant decay through distance, $\eta^{i n}$ represented the intra-island spatial auto-correlation, $\gamma^{\text {oc }}$ represented the association between species in occupancy and $\gamma^{\text {det }}$ represented the association between species in detection, $P(\theta>0 \mid$ data $)$ was the posterior probability that certain parameter was greater than 0

\begin{tabular}{llll}
\hline Parameter & Median & $95 \% \mathrm{Cl}$ & $p$ \\
\hline Fisher $\eta^{e x}$ & 2.48 & {$[0.976,4.74]$} & 0.00 \\
Marten $\eta^{e x}$ & -0.789 & {$[-1.91,-0.0558]$} & 0.032 \\
Fisher $\eta^{i n}$ & 0.0378 & {$[-0.232,0.328]$} & 0.80 \\
Marten $\eta^{i n}$ & 0.260 & {$[0.0551,0.444]$} & 0.02 \\
$\gamma_{o c}$ & 0.0870 & {$[-0.215,0.393]$} & 0.58 \\
$\gamma_{\text {det }}$ & -0.0122 & {$[-0.326,0.322]$} & 0.94 \\
\hline
\end{tabular}

process is a different question compared to the contribution of the process. However, Posterior distributions of negative potential functions in the fisher-marten system for different terms (Fig.4) reinforced the our finding that association had no significant contribution to the observed distribution pattern of the fisher-marten system. The fisher-marten system seemed to be dispersal/environment driven for fisher, while both dispersal/environment and intra-island spatial auto-correlation had similar levels of contribution for marten. Hence, competition is weak and had a minor contribution to the observed marten pattern which indicated a flipped mainland-island hypothesis where mainland functions as a sink rather than a source.

Posterior predictive checking showed all $6 \mathrm{p}$-values were greater than 0.05 indicating no systematic discrepancies between data and model predictions which suggested fitness of the fitted model (Fig.5). 
bioRxiv preprint doi: https://doi.org/10.1101/2020.08.05.238774; this version posted March 31, 2021. The copyright holder for this preprint (which was not certified by peer review) is the author/funder, who has granted bioRxiv a license to display the preprint in perpetuity. It is made available under aCC-BY-ND 4.0 International license.

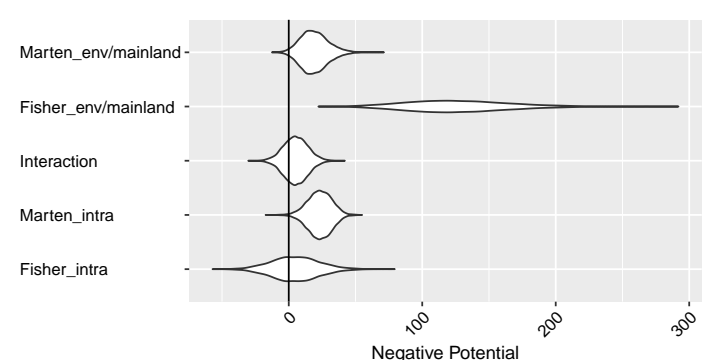

Figure 4. Posterior distribution of different terms in negative potential function for the Fisher-Marten system. Values represent the contribution of certain term in the negative potential function, note that we combined mainland-island and intercept which represented the overall environment.
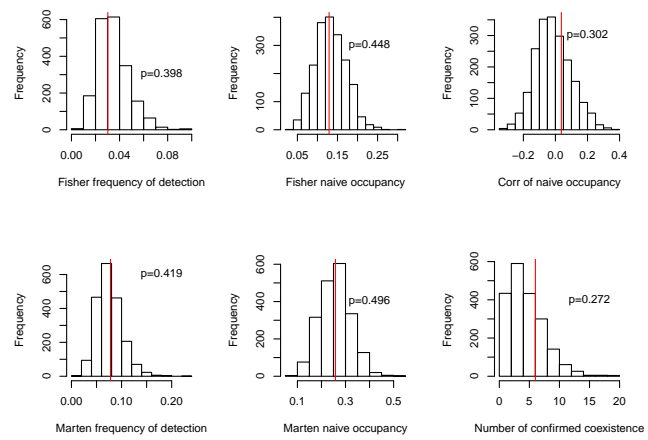

Figure 5.

Posterior predictive checking for the Fisher-Marten system and assessing model fitness. All $p$-values are large suggested that there were no conflict between data (red line) and predictions of our model according to the 6 statistics used thus a good fitness of the model.

Coyote-Fox System. $\log _{10}$ Bayes factors (log posterior odds of two models) for mainland-island and stepping stone models were estimated to be 18.3 (i.e. the posterior probability of mainland-island model was $10^{18}$ higher than the posterior probability of stepping stone model), hence data decisively supported the mainland-island rather than the stepping stone model following the recommended cutoff of 2 (Kass and Raftery, 1995). This result suggested that the spatial pattern of the system can be explained better by a mainland-island model rather than a stepping stone model. Further analysis was based on the Mainland-Island model.

Posterior estimates of model parameters (Table.3) indicated a significant positive distance dependency in fox but not coyote (Coyote: $\eta^{e x}=0.552, C I=[-0.378,1.69]$ Fox: $\left.\eta^{e x}=2.41, C I=[0.428,6.30]\right) \quad$ (Table.3). Meanwhile, posterior association in occupancy was estimated as positive but only weakly significant $\left(\gamma_{o c}=0.234, C I=\right.$ $[-0.041,0.53]$. The posterior probability of this parameter being positive was $p\left(\gamma_{o c}>0 \mid\right.$ data $\left.)=0.95\right)$, suggesting that despite dispersal/environment drivers, there may be evidence of a positive association between two species at grid level that needs further evaluation. These finding supported the hypothesis that coyote-fox system might not be fully explained by spatial factors and require further evaluation of their positive interactions. Notably, we detected a significant positive association in detections $\left(\gamma_{\text {det }}=0.427, C I=[0.211,0.646]\right)$ which might suggested a behavioral association.

Similar to the fisher-marten system, dispersal/environment also had the largest contribution to the observed pattern in
Table 3. Posterior estimates of model parameters in Coyote-Fox mainland-island system. $\eta^{e x}$ represented the distance dependency, $\eta^{e x}>0$ meant decay through distance, $\eta^{\text {in }}$ represented the intra-island spatial auto-correlation, $\gamma^{o c}$ represented the association between species in occupancy and $\gamma^{\text {det }}$ represented the association between species in detection, $P(\theta>0 \mid$ data $)$ was the posterior probability that certain parameter was greater than 0

\begin{tabular}{llll}
\hline Parameter & Median & $95 \% \mathrm{Cl}$ & $p$ \\
\hline Coyote $\eta^{e x}$ & 0.552 & {$[-0.3781 .69]$} & 0.24 \\
Fox $\eta^{e x}$ & 2.41 & {$[0.4286 .30]$} & 0.02 \\
Coyote $\eta^{i n}$ & 0.196 & {$[-0.01860 .426]$} & 0.08 \\
Fox $\eta^{\text {in }}$ & -0.0696 & {$[-0.3450 .207]$} & 0.60 \\
$\gamma_{o c}$ & 0.234 & {$[-0.04110 .528]$} & 0.1 \\
$\gamma_{\text {det }}$ & 0.427 & {$[0.2110 .646]$} & 0.00 \\
\hline
\end{tabular}

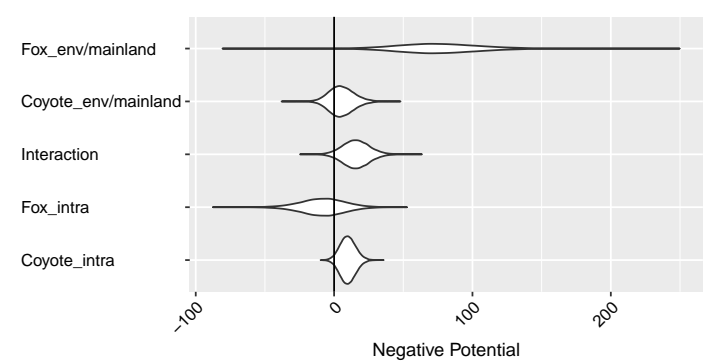

Figure 6. Posterior distribution of different terms in negative potential function for the Coyote-Fox system. Values represent the contribution of certain terms in the negative potential function, note that we combined mainland-island and intercept which represented the overall environment.

the coyote-fox system (Fig.6). However, in contrast with fisher-marten, both spatial auto-correlation and interspecific interaction seemed to have some importance (Fig.6) as also suggested by the hypothesis that there exist associations additive to spatial drivers.

Posterior predictive checking showed all 6 p-values were greater than 0.05 thus no systematic discrepancies between data and model predictions which suggested fitness of the fitted model (Fig.7).
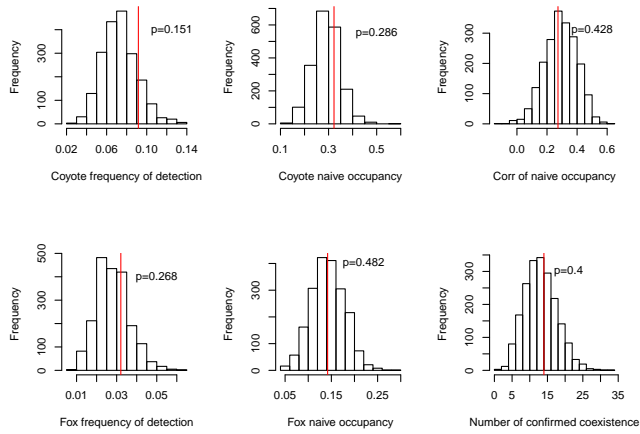

the Posterior predictive checking for All $p$-values are large suggested that there were no conflict between data (red line) and predictions of our model according to the 6 statistics used thus a good fitness of the model.

\section{Discussion}

We developed and tested a MRF-based, multispecies, spatially explicit, occupancy model which allowed evaluation of the relative contributions of spatial and life history drivers. 
bioRxiv preprint doi: https://doi.org/10.1101/2020.08.05.238774; this version posted March 31, 2021. The copyright holder for this preprint (which was not certified by peer review) is the author/funder, who has granted bioRxiv a license to display the preprint in perpetuity. It is made available under aCC-BY-ND 4.0 International license.

This model enables ecologists who conduct research on community structure to consider spatial and life history drivers jointly and explicitly. Though the model assumed patterns constant though time (single season), it is straightforward to extended it into a multiseason analysis. Compared with Bayesian network-based multispecies frameworks proposed by Kéry and Royle (2008), our method did not ask for a species to be the root of the network and allowed cycles in the network. Moreover, analysts can condition occupancy on another species to accommodate a single dominant competitor. Compared with Rota et al. (2016), our method had a better interpretation especially when a species network was large since in our method, interaction between species were modeled explicitly by auto-regression terms. Neither Kéry and Royle (2008) nor Rota et al. (2016) were spatially explicit. Partly because the graph represented the spatial correlation, it had no natural direction and could not be represented by a directed graph like Kéry and Royle (2008) did for species. Also when the number of potential outcomes was too large one cannot assign unique linear predictors for each pattern as in Rota et al. (2016). Markov random field modeling was used in quantifying interspecific interactions in Harris (2016) and can help identify interactions between species when controlling for environment and other confounding interactions (e.g. apparent competition where A, B both interact with C while no interaction occurs between $A$ and $B$ ) (Blanchet et al., 2020).

Our results on two pairs of plausibly competing species as components of the meso-carnivore community also showed that community structure reflected drivers associated with two broad theoretical paradigms. First, we detected positive intra-island spatial-autocorrelation for 2 species out of 4 . This spatial autocorrelation term makes sites no longer exchangable even when distance to mainland was controlled. Spatial autocorrelation also had different strengths for the four species considered, which means species were not exchangeable even when considering spatial processes. Coyotes and foxes had different strengths of dependence on mainland distance, likely due to different dispersal ability, i.e. coyotes (which are larger) likely can disperse farther than foxes and, thus have weaker distance dependency.

We detected an unexpected opposite direction of mainland distance dependency on occupancy of marten. The fisher-marten system provides a possible example where the mainland serves as sink rather than source for a species from a metapopulation point of view. This spatial pattern was recently verified independently for martens using genetic techniques (e.g. Smith et al. (2020), in review). A more general meta-community framework should be used when considering island or island-like systems. If two species follow similar dispersal patterns but need to partition spatially we should expect closer islands to be more likely occupied by one of the species than the further islands and partitioning should happen on islands with similar, nearby distances to mainland (i.e. perpendicular to the mainland-island dispersion direction). However, we observed similar levels of co-absence on islands regardless of their distances to mainland, i.e. martens were not occupying nearby islands regardless of fisher presence. Meanwhile, the partitioning of fisher and marten happened in parallel (distance dependency) rather than independently of distance (i.e., competition) to the mainland-island dispersal direction which was different from what we would expect based on hypothesis 1 (i.e., distance dependency with competition). We did not observed fisher and marten occupying sites closer to mainland or partitioning at site level. The fisher-marten system appeared to better conform to hypothesis 2, because fisher dispersal direction appeared to be from the mainland to the islands, while marten dispersal appeared to be from the islands to the mainland. However, these results were solely from distribution data and additional evidence from genetics (e.g., Smith et al. (2020), in review), movement measurements, or behavior etc. would be needed to further support this argument.

For the coyote-fox system, we observed that spatial auto-correlation had a strong influence on coyote distribution. This may be due to the relatively small size of the islands compared to coyote home ranges. Typical coyote home ranges were $\sim 10 \mathrm{~km}^{2}$ (Mills and Knowlton, 1991; Hibler, 1977) which is approximately the size of the largest islands in APIS. Home range size reported for red fox was smaller and in $1 \sim 10 \mathrm{~km}^{2}$ scale (Ables, 1969; Dekker et al., 2001; Trewhella et al., 1988) and was smaller in size than some individual islands in APIS. Together with mainland distance-dependency, spatial correlation patterns of coyote and fox were consistent with our knowledge of their movement ability, i.e., coyotes have stronger dispersal ability and larger home ranges, thus, weaker distance-dependency and stronger intra-island spatial autocorrelation compared with foxes. Patterns of coyotes and foxes demonstrate that species are not interchangeable (Island Biogeography Theory) and that distance-dependency is modified by life history characteristics.

The fisher-marten system also had a spatial auto-correlation effect. Furnas et al. (2017) reported a meta-analysis on home range sizes in California, USA. Their results showed that home ranges of female fishers varied and were approximately $6 \mathrm{~km}^{2}$ within $20 \mathrm{~km}$ of the coast while approximately $13 \mathrm{~km}^{2}$ at $120 \mathrm{~km}$ from the coast. Male fishers had home range sizes that varied from $12 \mathrm{~km}^{2}$ at $20 \mathrm{~km}$ to approximately $27 \mathrm{~km}^{2}$ at $120 \mathrm{~km}$ to the coast. We did not detect strong intra-island spatial auto correlation in fishers, which may indicate a relatively small home range for these animals on islands compared to other studies, which was consistent with prior knowledge that fisher home ranges decline when close to coasts (Powell, 1982; Yaeger, 2005). Studies of marten home ranges in Canada indicated their home ranges can vary from $10 \sim 100 \mathrm{~km}^{2}$ (Smith and Schaefer, 2002), we also detected spatial auto-correlation for martens. Further home range and movement studies may be needed to confirm our findings based on distributions on these islands. Studies in Canada on interaction between coyotes and foxes showed that they typically partition through habitat use. But this pattern depended heavily on prey abundance (Theberge and Wedeles, 1989). Evidence also showed coyotes may aggressively kill red foxes (Gese et al., 1996). The positive correlation between foxes and coyotes may suggest that foxes trade off predation risk for prey availablity in a prey-limited system. Given the relatively small scale of this island system, environment was rather homogeneous and the only environmental variation we were able to obtain was distance to mainland which is, itself, spatial. The model has the ability to incorporate additional environmental variation but measurements are difficult to obtain. To better understand the system with the proposed model detailed environmental variables like prey biomass should be measured to further explain the positive correlation pattern. Further study should be conducted to further evaluate these spatial distributions. 
bioRxiv preprint doi: https://doi.org/10.1101/2020.08.05.238774; this version posted March 31, 2021. The copyright holder for this preprint (which was not certified by peer review) is the author/funder, who has granted bioRxiv a license to display the preprint in perpetuity. It is made available under aCC-BY-ND 4.0 International license.

\section{Conclusion}

We implemented a MRF based multi-specific occupancy model that can account for both spatial auto-correlations and inter-specific interactions simultaneously. We used this technique in a case-study on two pairs of presumably competing species in the Apostle Island National Lakeshore (Wisconsin, USA). We detected a partitioning pattern of fisher and marten occupancy, which can be explained by a flipped source-sink pattern in the islands. However, additional evidence from movement data or genetics might be needed to further confirm this observation. We detected a positive association among coyote and fox, which, is different from studies on mainland systems and deserves further study.

\section{Acknowledgment}

We appreciate helpful discussions with Dr. Claudia Solís-Lemus from Wisconsin Institute for Discovery during manuscript preparation and data collection support from the National Park Service. We thank the many students and staff that have participated in data collection. Y.S. acknowledges Jialong Jiang and Binxu Wang's discussion during model and algorithm development. This research was funded by the United States National Park Service (USDI) through the Apostle Islands National Lakeshore (GLNF CESU Agreement P14AC01180), NASA Earth and Space Science Fellowship (Grant number NNX16AO61H). Additional funding came from the University of Wisconsin-Madison's Beers-Bascom professorship and the UW-Madison department of Forest and Wildlife Ecology's A. W. Schorger fund to TVD and Sigurd Olson Professorship in the Department of Natural Resources at Northland College to E.O. We greatly appreciate the helpful feedback from two anonymous reviewers on the early draft of this manuscript.

\section{References}

Ables, E. D. (1969). Home-range studies of red foxes (vulpes vulpes). Journal of Mammalogy, 50(1):108-120.

Allen, M. L., Evans, B. E., Wheeler, M. E., Mueller, M. A., Pemble, K., Olson, E. R., Van Stappen, J., and Van Deelen, T. R. (2017). Survey techniques, detection probabilities, and the relative abundance of the carnivore guild on the apostle islands (2014-2016). arXiv preprint arXiv:1703.10726.

Bates, D. and Maechler, M. (2019). Matrix: Sparse and Dense Matrix Classes and Methods. $\mathrm{R}$ package version 1.2-17.

Becker, O. M. and Karplus, M. (1997). The topology of multidimensional potential energy surfaces: Theory and application to peptide structure and kinetics. The Journal of chemical physics, 106(4):1495-1517.

Blanchet, F. G., Cazelles, K., and Gravel, D. (2020). Co-occurrence is not evidence of ecological interactions. Ecology Letters.

Chalmond, B. (1989). An iterative gibbsian technique for reconstruction of m-ary images. Pattern recognition, 22(6):747-761.

Cipra, B. A. (1987). An introduction to the ising model. The American Mathematical Monthly, 94(10):937-959

Cottenie, K. (2005). Integrating environmental and spatial processes in ecological community dynamics. Ecology letters, 8(11):1175-1182.

Cressie, N. (1992). Statistics for spatial data. Terra Nova, 4(5):613-617.

Dekker, J. J., Stein, A., and HeitkoĖnig, I. M. (2001). A spatial analysis of a population of red fox (vulpes vulpes) in the dutch coastal dune area. Journal of Zoology, 255(4):505-510.

Descombes, X., Morris, R. D., Zerubia, J., and Berthod, M. (1999). Estimation of markov random field prior parameters using markov chain monte carlo maximum likelihood. IEEE Transactions on Image Processing, 8(7):954-963.

Dray, S., Legendre, P., and Peres-Neto, P. R. (2006). Spatial modelling: a comprehensive framework for principal coordinate analysis of neighbour matrices (pcnm). ecological modelling, 196(3-4):483-493.

Eddelbuettel, D. and François, R. (2011). Rcpp: Seamless R and $C_{++}$integration. Journal of Statistical Software, 40(8):1-18.

Eddelbuettel, D. and Sanderson, C. (2014). Rcpparmadillo: Accelerating $r$ with high-performance c++ linear algebra. Computational Statistics and Data Analysis, 71:1054-1063.

Epskamp, S. (2015). IsingSampler: Sampling Methods and Distribution Functions for the Ising Model. R package version 0.2 .
Ezaki, T., Watanabe, T., Ohzeki, M., and Masuda, N. (2017). Energy landscape analysis of neuroimaging data. Philosophical Transactions of the Royal Society A: Mathematical, Physical and Engineering Sciences, 375(2096):20160287.

Ferguson, A. L., Mann, J. K., Omarjee, S., Ndung'u, T., Walker, B. D., and Chakraborty, A. K. (2013). Translating hiv sequences into quantitative fitness landscapes predicts viral vulnerabilities for rational immunogen design. Immunity, 38(3):606-617.

Furnas, B., Landers, R., Callas, R., and Matthews, S. (2017). Estimating population size of fishers (pekania pennanti) using camera stations and auxiliary data on home range size. Ecosphere, 8(3):e01747.

Gastauer, M. and Meira-Neto, J. A. A. (2014). Interactions, environmental sorting and chance: phylostructure of a tropical forest assembly. Folia Geobotanica, 49(3):443-459.

Gelman, A., Carlin, J. B., Stern, H. S., Dunson, D. B., Vehtari, A., and Rubin, D. B. (2013). Bayesian data analysis. Chapman and Hall/CRC.

Gelman, A., Meng, X.-L., and Stern, H. (1996). Posterior predictive assessment of model fitness via realized discrepancies. Statistica sinica, pages $733-760$.

Gese, E. M., Stotts, T. E., and Grothe, S. (1996). Interactions between coyotes and red foxes in yellowstone national park, wyoming. Journal of Mammalogy, 77(2):377-382.

Göthe, E., Angeler, D. G., and Sandin, L. (2013). Metacommunity structure in a small boreal stream network. Journal of Animal Ecology, 82(2):449-458.

Grinnell, J. (1917). The niche-relationships of the california thrasher. The Auk, 34(4):427-433

Hanski, I. (1983). Coexistence of competitors in patchy environment. Ecology, 64(3):493-500.

Harris, D. J. (2016). Inferring species interactions from co-occurrence data with markov networks. Ecology, 97(12):3308-3314.

Hastings, W. K. (1970). Monte carlo sampling methods using markov chains and their applications. Biometrika, 57:97-109.

Hepler, S. A., Erhardt, R., and Anderson, T. M. (2018). Identifying drivers of spatial variation in occupancy with limited replication camera trap data. Ecology.

Hibler, S. J. (1977). Coyote movement patterns with emphasis on home range characteristics. Master's thesis, Utah State University.

Hubbell, S. P. (2001). The unified neutral theory of biodiversity and biogeography (MPB-32). Princeton University Press.

Hughes, J., Haran, M., and Caragea, P. C. (2011). Autologistic models for binary data on a lattice. Environmetrics, 22(7):857-871.

Hutchinson, G. (1957). The multivariate niche. In Cold Spring Harbor Symposia on Quantitative Biology, volume 22, pages 415-421.

Ibáñez, M. V. and Simó, A. (2003). Parameter estimation in markov random field image modeling with imperfect observations. a comparative study. Pattern recognition letters, 24(14):2377-2389.

Ising, E. (1925). Contribution to the theory of ferromagnetism. Z. Phys., 31:253-258.

Kass, R. E. and Raftery, A. E. (1995). Bayes factors. Journal of the American Statistical Association, 90(430):773-795.

Kéry, M. and Royle, J. A. (2008). Hierarchical bayes estimation of species richness and occupancy in spatially replicated surveys. Journal of Applied Ecology, 45(2):589-598.

Kéry, M. and Schmidt, B. (2008). Imperfect detection and its consequences for monitoring for conservation. Community Ecology, 9(2):207-216.

Koller, D. and Friedman, N. (2009). Probabilistic graphical models: principles and techniques. MIT press.

Lasky, J. R., Keitt, T. H., Weeks, B. C., and Economo, E. P. (2017). A hierarchical model of whole assemblage island biogeography. Ecography, 40(8):982-990.

Leibold, M. A., Holyoak, M., Mouquet, N., Amarasekare, P., Chase, J. M., Hoopes, M. F. Holt, R. D., Shurin, J. B., Law, R., and Tilman, D. (2004). The metacommunity concept: a framework for multi-scale community ecology. Ecology letters, 7(7):601-613.

Levy, R. M., Haldane, A., and Flynn, W. F. (2017). Potts hamiltonian models of protein co-variation, free energy landscapes, and evolutionary fitness. Current opinion in structural biology, 43:55-62.

Logue, J. B., Mouquet, N., Peter, H., Hillebrand, H., and Group, M. W. (2011). Empirical approaches to metacommunities: a review and comparison with theory. Trends in ecology and evolution, 26(9):482-491.

Lotka, A. J. (1910). Contribution to the theory of periodic reactions. The Journal of Physical Chemistry, 14(3):271-274.

Lynch, S. M. and Western, B. (2004). Bayesian posterior predictive checks for complex models. Sociological methods \& research, 32(3):301-335.

MacArthur, R. H. and Wilson, E. O. (2001). The theory of island biogeography, volume 1. Princeton university press.

MacKenzie, D. I., Nichols, J. D., Hines, J. E., Knutson, M. G., and Franklin, A. B. (2003) Estimating site occupancy, colonization, and local extinction when a species is detected imperfectly. Ecology, 84(8):2200-2207.

Meyer, K. (2017). Islands in a Sea of Mud: Insights From Terrestrial Island Theory for Community Assembly on Insular Marine Substrata, volume 76, book section 1, pages 1-40. Elsevier.

Mills, L. S. and Knowlton, F. F. (1991). Coyote space use in relation to prey abundance. Canadian Journal of Zoology, 69(6):1516-1521.

Morcos, F., Schafer, N. P., Cheng, R. R., Onuchic, J. N., and Wolynes, P. G. (2014). Coevolutionary information, protein folding landscapes, and the thermodynamics of natural selection. Proceedings of the National Academy of Sciences, 111(34):12408-12413.

Murray, I., Ghahramani, Z., and MacKay, D. (2012). Mcmc for doubly-intractable distributions. arXiv preprint arXiv:1206.6848.

Møller, J., Pettitt, A. N., Reeves, R., and Berthelsen, K. K. (2006). An efficient markov chain monte carlo method for distributions with intractable normalising constants. Biometrika, 93(2):451-458.

Okabe, A., Boots, B., Sugihara, K., and Chiu, S. N. (2009). Spatial tessellations: concepts and applications of Voronoi diagrams, volume 501. John Wiley \& Sons.

Osogami, T. (2017). Boltzmann machines and energy-based models. arXiv preprint arXiv: 1708.06008 
bioRxiv preprint doi: https://doi.org/10.1101/2020.08.05.238774; this version posted March 31, 2021. The copyright holder for this preprint (which was not certified by peer review) is the author/funder, who has granted bioRxiv a license to display the preprint in perpetuity. It is made available under aCC-BY-ND 4.0 International license.

Pearl, J. (1995). Causal diagrams for empirical research. Biometrika, 82(4):669-688.

Pfeuty, P. (1970). The one-dimensional ising model with a transverse field. ANNALS of Physics, 57(1):79-90

Plummer, M., Best, N., Cowles, K., and Vines, K. (2006). Coda: Convergence diagnosis and output analysis for mcmc. R News, 6(1):7-11.

Powell, R. A. (1982). The fisher: life history, ecology, and behavior. University of Minnesota Press.

Propp, J. G. and Wilson, D. B. (1996). Exact sampling with coupled markov chains and applications to statistical mechanics. Random Structures \& Algorithms, 9(1-2):223-252.

R Core Team (2019). R: A Language and Environment for Statistical Computing. R Foundation for Statistical Computing, Vienna, Austria.

Raftery, A. E., Newton, M. A., Satagopan, J. M., and Krivitsky, P. N. (2006). Estimating the integrated likelihood via posterior simulation using the harmonic mean identity. Memorial Sloan-Kettering Cancer Center, Dept. of Epidemiology \& Biostatistics Working Paper Series.

Rota, C. T., Ferreira, M. A., Kays, R. W., Forrester, T. D., Kalies, E. L., McShea, W. J., Parsons, A. W., and Millspaugh, J. J. (2016). A multispecies occupancy model for two or more interacting species. Methods in Ecology and Evolution, 7(10):1164-1173.

Saporetti-Junior, A. W., Schaefer, C. E. G. R., de Souza, A. L., Soares, M. P., Araújo, D. S. D., and Meira-Neto, J. A. A. (2012). Influence of soil physical properties on plants of the mussununga ecosystem, brazil. Folia Geobotanica, 47(1):29-39.

Shekhar, K., Ruberman, C. F., Ferguson, A. L., Barton, J. P., Kardar, M., and Chakraborty, A. K. (2013). Spin models inferred from patient-derived viral sequence data faithfully describe hiv fitness landscapes. Phys. Rev. E, 88:062705

Shurin, J. B., Cottenie, K., and Hillebrand, H. (2009). Spatial autocorrelation and dispersal limitation in freshwater organisms. Oecologia, 159(1):151-159.

Smith, A. C. and Schaefer, J. A. (2002). Home-range size and habitat selection by american marten (martes americana) in labrador. Canadian Journal of Zoology, 80(9):1602-1609.

Smith, M. M., Gilbert, J. H., E.R.Olson, Scribner, K., Van Deelen, T., Van Stappen, J., Williams, B., Woodford, J., and Pauli, J. (2020). A recovery network leads to the natural recolonization of an archipelago and a potential trailing edge refuge (in review). Revision submitted to Ecological Applications, December 82020.

Theberge, J. B. and Wedeles, C. H. (1989). Prey selection and habitat partitioning in sympatric coyote and red fox populations, southwest yukon. Canadian Journal of Zoology, 67(5):1285-1290.

Tilman, D. (1985). The resource-ratio hypothesis of plant succession. The American Naturalist, 125(6):827-852.

Trewhella, W., Harris, S., and McAllister, F. (1988). Dispersal distance, home-range size and population density in the red fox (vulpes vulpes): a quantitative analysis. Journal of Applied Ecology, pages 423-434.

Vanmarcke, E. (2010). Random fields: analysis and synthesis. World Scientific.

Volkov, I., Banavar, J. R., Hubbell, S. P., and Maritan, A. (2003). Neutral theory and relative species abundance in ecology. Nature, 424(6952):1035.

Volterra, V. (1928). Variations and fluctuations of the number of individuals in animal species living together. ICES Journal of Marine Science, 3(1):3-51.

Wei, Z. and Li, H. (2007). A markov random field model for network-based analysis of genomic data. Bioinformatics, 23(12):1537-1544.

West, R., Paskov, H. S., Leskovec, J., and Potts, C. (2014). Exploiting social network structure for person-to-person sentiment analysis. Transactions of the Association for Computational Linguistics, 2:297-310.

Wolters, M. A. (2017). Better autologistic regression. Frontiers in Applied Mathematics and Statistics, 3(24).

Yaeger, J. S. (2005). Habitat at fisher resting sites in the Klamath Province of northern California. PhD thesis, Humboldt State University Arcata, California.

Zhu, J., Huang, H.-C., and Wu, J. (2005). Modeling spatial-temporal binary data using markov random fields. Journal of Agricultural, Biological, and Environmental Statistics, 10(2):212. 


\section{Supplementary Materials}

\section{Supplementary Note S1: Stepping Stone Graph}

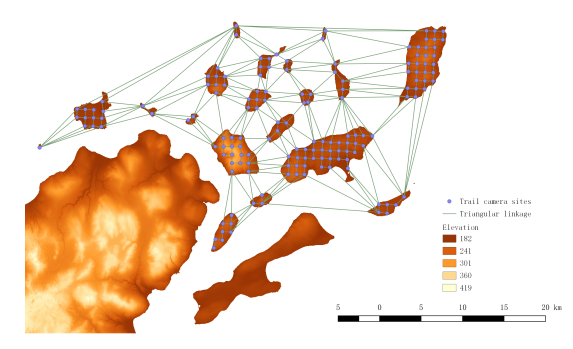

Figure S1. Graph used in Stepping stone model

\section{Supplementary Note S2: MH Algorithm and Bayes factor}

Before Moller's work in 2006, Bayesian inference on MRF models was precluded because the intractable normalizing constant is a function of parameters of interest. Moller proposed an auxiliary variable based on the Metropolis-Hastings ratio (Møller et al., 2006). A further development due to Murray et al. (2012) is called the single parameter change method. In our case, we follow Murray et al. (2012) by sampling an auxiliary variable $X$ to follow the MRF distribution whose parameters are proposed $\theta^{\prime}$. Together with imperfect detection, the Metropolis-Hastings ratio is given by eqn.S1.

$$
M H\left(\theta^{\prime} \mid \theta\right)=\frac{\pi\left(\theta^{\prime}\right) p(y \mid Z) q_{\theta^{\prime}}(Z) q_{\theta}(X)}{\pi(\theta) p(y \mid Z) q_{\theta}(Z) q_{\theta^{\prime}}(X)}
$$

The sample can be drawn using the Coupling From the Past algorithm (CFTP Propp and Wilson (1996)) or long enough Gibbs chain for approximation. We tested the difference between using a perfect sample taken by CFTP algorithm and a Gibbs sample in the single parameter exchange algorithm. Results showed if iteration for Gibbs is large enough (e.g. > $150)$ the posterior distribution sampled by these two methods were essentially the same (see example of Fig.S3). CFTP and Gibbs were implemented in $\mathrm{R}$ and $\mathrm{C}++$ modified from the $R$ package IsingSampler (Epskamp, 2015) with help of RcppArmadillo (Eddelbuettel and Sanderson, 2014) and sparse matrix $\mathrm{C}++$ class provided by $\mathrm{R}$ package Matrix (Bates and Maechler, 2019) and Armadillo optimized for the sparse graph as we have (open sourced as $R$ package SparseIsingSampler available on GitHub).

Posterior sample of $\mathbf{Z}$ will also be taken using a Gibbs algorithm, with fully conditional odds of being +1 as

$$
\begin{aligned}
\frac{P\left(Z_{k i}=+1 \mid Z_{-k i}, y\right)}{P\left(Z_{k i}=-1 \mid Z_{-k i}, y\right)}= & \frac{p\left(y_{1 i .}, y_{2 i . . .} y_{w i .} \mid Z_{k i}=1, z_{. i}\right)}{p\left(y_{1 i .}, y 2 i \ldots . y_{w i .} \mid Z_{k i}=-1, z_{. i}\right)} \\
& \times \frac{\exp \left(X_{i} \beta_{k}+\sum_{j \in n(i)} \gamma_{i j}^{o c} Z_{j}\right)}{\exp \left(-X_{i} \beta_{k}-\sum_{j \in n(i)} \gamma_{i j}^{o c} Z_{j}\right)}
\end{aligned}
$$

To calculate the Bayes factor, we need to calculate the likelihood of each sample then using the harmonic rule (Raftery et al., 2006). To calculate the likelihood, we take a sample $\mathbf{Y}$ from a pre-specified parameter setting $\phi$, the ratio of normalizing constant $C(\theta)$ and $C(\phi)$ can be calculated as the expectation: $E q_{\theta-\phi}(\mathbf{Y})$. We can calculate log likelihood added by $-\log (C(\phi))$ which is intractable. However, by choosing the same $\phi$ for two competing models, we can calculate BF of two models by canceling out the intractable constant induced by $\phi$.

\section{Supplementary Note S3: Simulation}

Table S1. Simulation setting for regular lattice

\begin{tabular}{llll}
\hline Parameter & Competition & No interaction & Sorting \\
\hline$\beta_{1}$ & 0.5 & 0.5 & 0.3 \\
$\beta_{2}$ & 0.5 & -0.5 & 0.3 \\
$\eta^{i n}$ & 0.25 & 0.25 & 0.25 \\
$\gamma_{o c}$ & -0.3 & 0 & 0.3 \\
$\gamma_{\text {det }}$ & -0.2 & 0 & 0.2 \\
\hline
\end{tabular}

Table S2. Simulation setting for APIS

\begin{tabular}{llll}
\hline Parameter & Competition & No interaction & Sorting \\
\hline$\eta_{1}^{\text {ex }}$ & 1 & 1 & 0.3 \\
$\eta_{2}^{\text {ex }}$ & 1 & -1 & 0.3 \\
$\eta^{i n}$ & 0.2 & 0.2 & 0.2 \\
$\gamma_{o c}$ & -0.3 & 0 & 0.25 \\
$\gamma_{\text {det }}$ & -0.2 & 0 & 0.2 \\
\hline
\end{tabular}

\section{Supplementary Note S4: MCMC Diagnostic}

We showed the trace plot and auto-correlation function of the interspecific interaction strength in occupancy $\gamma_{o c}$ as an example diagnostic

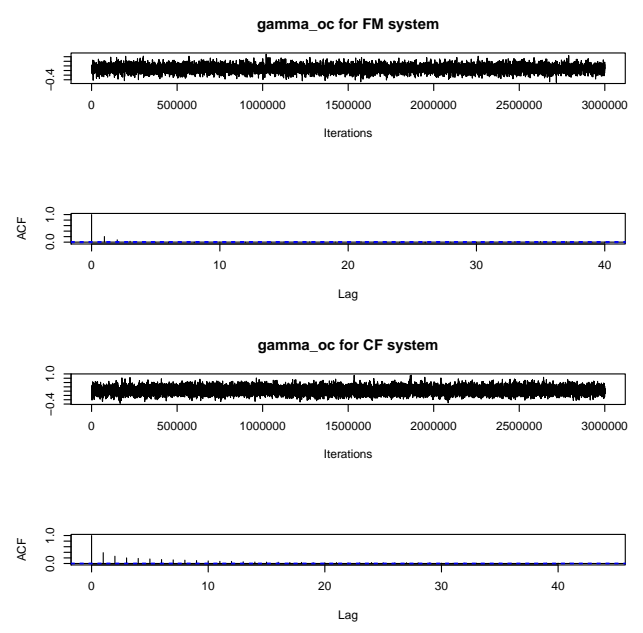

Figure S2. MCMC for $\gamma_{o c}$ in FM and CF

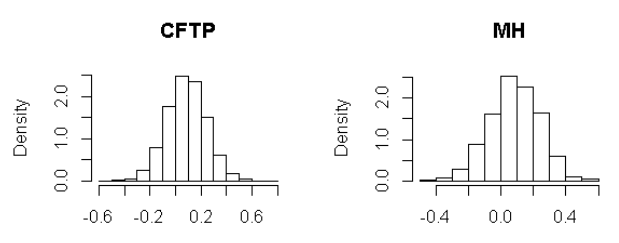

Figure S3. One example of same task using CFTP and MH, KS test had p-value of 0.9383 
bioRxiv preprint doi: https://doi.org/10.1101/2020.08.05.238774; this version posted March 31, 2021. The copyright holder for this preprint (which was not certified by peer review) is the author/funder, who has granted bioRxiv a license to display the preprint in perpetuity. It is made available under aCC-BY-ND 4.0 International license.

\section{Supplementary Note S5: Release of Prior on Detection Rate}

Again it is not necessary to set this prior in a more general setting. But in the APIS case study, if we release this prior on the intercept of detection rate, the posterior had multiple modes for fisher and marten model, due to the fact that fisher's low naive detection can be due to both low occupancy or low detection, these results won't influence our conclusion about fisher and marten system but rather influence the numerical result of spatial auto correlation strength. This will cause a very large and unrealistic mainland-island strength in fisher S4.

Fisher mainland-island

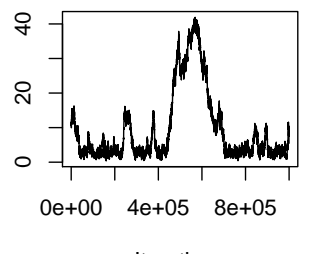

Iterations

\section{Fisher mainland-island}

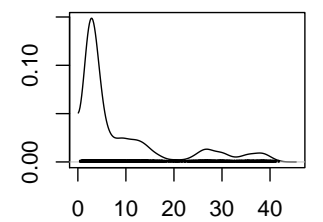

$\mathrm{N}=10000$ Bandwidth $=1.174$

Figure S4. Multiple modes in fisher's mainland-island strength if release the prior 